\title{
THE EMISSION-LINE SPECTRA OF MAJOR MERGERS: EVIDENCE FOR SHOCKED OUTFLOWS*
}

\author{
Kurt T. Soto ${ }^{1}$, C. L. Martin ${ }^{1}$, M. K. M. Prescott ${ }^{1}$, And L. Armus $^{2}$ \\ ${ }^{1}$ Physics Department, University of California, Santa Barbara, CA 93106-9530, USA \\ 2 Spitzer Science Center, California Institute of Technology, Pasadena, CA, USA \\ Received 2012 April 10; accepted 2012 July 29; published 2012 September 6
}

\begin{abstract}
Using a spectral decomposition technique, we investigate the physical origin of the high-velocity emission-line gas in a sample of 39 gas-rich, ultraluminous infrared galaxy mergers. Regions with shock-like excitation were identified in two kinematically distinct regimes, characterized by broad $\left(\sigma>150 \mathrm{~km} \mathrm{~s}^{-1}\right)$ and narrow linewidths $\left(\sigma \leqslant 150 \mathrm{~km} \mathrm{~s}^{-1}\right)$. Here, we investigate the physical origin of the broad emission, which we show is predominantly excited by shocks with velocities of $200-300 \mathrm{~km} \mathrm{~s}^{-1}$. Considering the large amount of extinction in these galaxies, the blueshift of the broad emission suggests an origin on the near side of the galaxy and therefore an interpretation as a galactic outflow. The large spatial extent of the broad, shocked emission component is generally inconsistent with an origin in the narrow-line region of an active galactic nucleus. The kinetic energy in the mass loss as well as the luminosity of the emission lines is consistent with the fraction of the supernova energy attributed to these mechanisms by shocked stellar winds. Since some shocks can be recognized in moderately high resolution, integrated spectra of nearby ultraluminous starbursts, the spectral fitting technique introduced in Soto \& Martin may therefore be used to improve the accuracy of the physical properties measured for high-redshift galaxies from their (observed frame) infrared spectra.
\end{abstract}

Key words: galaxies: active - galaxies: evolution - galaxies: formation - galaxies: starburst

Online-only material: color figures

\section{INTRODUCTION}

Ultraluminous infrared galaxies (ULIRGs) are some of the most powerful galaxies in the local universe, with $\log \left(L_{\mathrm{IR}} / L_{\odot}\right)>12$. Many ULIRGs exhibit disturbed morphologies indicative of major mergers (Borne et al. 1999). As the merger progresses, the tidal interactions between the galaxies are believed to drive gas inflow capable of fueling both central starbursts and an active galactic nucleus (AGN) (Toomre \& Toomre 1972; Sanders et al. 1986; Springel et al. 2005; Hopkins et al. 2008).

Models of major mergers also predict an early phase of supernova feedback followed by the emergence of an AGN (Springel et al. 2005; Hopkins et al. 2005; Hopkins 2012). The starburst-driven outflows found in $75 \%-80 \%$ of ULIRGs provide evidence for this early supernova feedback phase (Rupke et al. 2002; Martin 2005). In these galaxies, starburst-driven outflows produce interstellar absorption lines blueshifted a few hundred $\mathrm{km} \mathrm{s}^{-1}$ relative to the systemic velocity set by molecular gas and stars (Armus et al. 1990; Heckman et al. 1990; Martin 2005, 2006; Rupke et al. 2005a, 2005b, 2005c; Rupke \& Veilleux 2005). AGN-driven outflows, in contrast, have only been found in the small subset of ULIRGs with Seyfert 1 nuclei (Rupke et al. 2005a), possibly indicating that this mechanism takes over at a stage following the merger-triggered starburst-driven outflows. These mechanisms for removing gas, along with star formation and black hole fueling, provide the last step in transforming ULIRGs into field elliptical galaxies (Dasyra et al. 2006) — a process already suggested by stellar velocity dispersions that exceed stellar rotation speed and surface brightness profile fit by $r^{1 / 4}$ laws (Genzel et al. 2001).

\footnotetext{
* The data presented herein were obtained at the W. M. Keck Observatory, which is operated as a scientific partnership among the California Institute of Technology, the University of California, and the National Aeronautics and Space Administration. The Observatory was made possible by the generous financial support of the W. M. Keck Foundation.
}

The high star formation rates (SFRs) common among $z \sim 2-3$ galaxies are thought to be fueled by steady gas accretion instead of mergers (Daddi et al. 2007; Shapiro et al. 2008; Noguchi 1999), but mergers may play a pivotal role in the formation of galactic spheroids even at these redshifts (Hopkins et al. 2011). Regardless of how these galaxies get their gas, comparable SFRs are only found among ULIRGs and the Lyman-Break Analogs (Overzier et al. 2008, 2009a, 2009b, 2011; Heckman et al. 2011) in the local universe, so these environments provide the best local laboratories for studying the feedback processes that shape the evolution of high-redshift galaxies.

Given the importance of incorporating feedback from massive stars and AGNs into galaxy formation simulations, simultaneously mapping out the excitation and gas kinematics in these extreme, local environments is of broad interest (Heckman et al. 1987, 1990; Armus et al. 1990; Murray et al. 2005; Veilleux et al. 2009). The emission-line spectrum of shocked regions, for example, is easily distinguished from the spectrum of gas photoionized by massive stars but quite similar to the spectrum of gas photoionized by an AGN. Because the size of the narrow-line region (NLR) powered by an AGN is limited by the AGN luminosity (Bennert et al. 2006a, 2006b; Greene et al. 2011), one strategy for breaking this degeneracy between excitation mechanisms is to resolve the location of the gas emitting the shock-like spectrum (Monreal-Ibero et al. 2006; Rich et al. 2011; Gonçalves et al. 2010; Soto \& Martin 2012, hereafter Paper I). Large velocity dispersions provide another way to identify regions with strong feedback from star formation and active nuclei, gravitational instability, and/or streams of recently accreted material (Genzel et al. 2011; Law et al. 2009; Schreiber et al. 2006), and the Doppler shifts of broad emission lines can be used to distinguish shocks generated by galactic winds and infall from either cold streams or tidally stripped gas. 


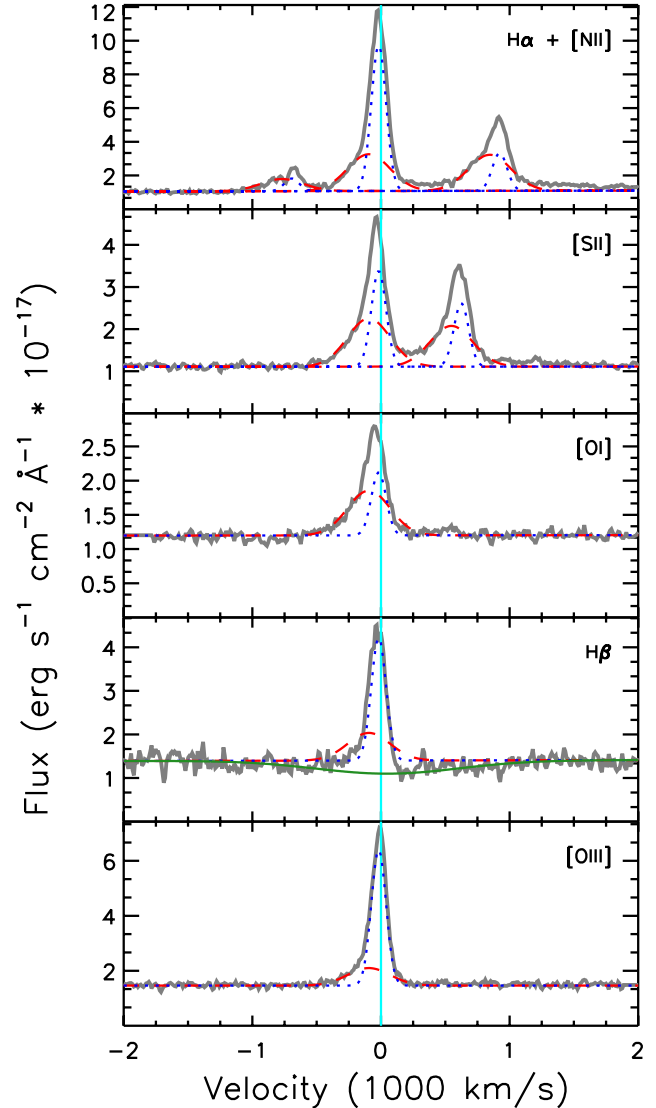

Figure 1. In the above plots, the gray line is the data; the dotted blue and dashed red lines show the different components in each transition, with each line type representing the same kinematics in each transition; the green line represents the continuum with an $\mathrm{H} \beta$ absorption component; and the vertical cyan line represents the systemic velocity. Example of emission-line fit from Paper I; the line profiles of the different transitions were fit with common kinematic components of varying flux. This figure demonstrates the clear variations in the line profiles for each transition, i.e., the blueshifted wing on $\mathrm{H} \alpha$, the nearly triangular line profile of $[\mathrm{N}$ II] $\lambda 6583$, and the very low intensity blue wing on $[\mathrm{O} \mathrm{III}] \lambda 5007$. Using this fitting method, we are able to deconstruct the line profile and examine the excitation mechanism for all of the spectral components that show the same kinematics.

(A color version of this figure is available in the online journal.)

To provide a comprehensive examination of the location and speeds of shocks in ULIRGs, we mapped the emissionline ratios across 39 local $(z=0.043-0.152)$ ULIRGs in velocity and along one spatial direction with the Keck Echellette Spectrograph and Imager (ESI). While these long-slit spectra lack the complete spatial coverage of an integral field unit (IFU), they offer more sensitivity and broader spectral coverage than IFU observations. In Paper I, we identified regions with shock-like line ratios. Following a brief summary of the sample and observations in Section 2 of this paper, we present the velocity dispersion and Doppler shift of the shocked regions in Section 3 and argue that outflows are the primary explanation for shocked regions with broad $\left(\sigma>150 \mathrm{~km} \mathrm{~s}^{-1}\right)$ emission lines. In Section 4, we discuss the origin of the outflows and estimate the outflow properties. Finally, we discuss the impact of shocked gas on the integrated spectrum in Section 5.1.

\section{OBSERVATIONS AND REDUCTIONS}

This study examines the measurements presented in Paper I of 39 ULIRGs at various stages of merging chosen from the IRAS 2 Jy survey (Murphy et al. 1996). These local galaxies span

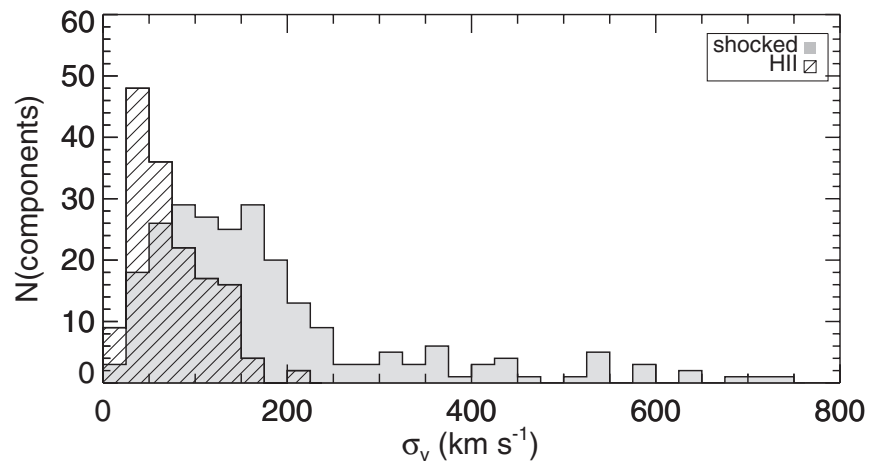

Figure 2. In the above histograms, we present the distributions of line width for components identified as "shock-like" and those identified as "H II-like." Nearly all components with $\mathrm{H}$ II-like line ratios have $\sigma_{v}<150 \mathrm{~km} \mathrm{~s}^{-1}$. The distribution of components identified as shock-like has a tail out to large line widths. (Of the 430 components measured, 34 components are not shown here because the measurement uncertainties did not place them uniquely in one of these two excitation classes.)

the redshifts $z=0.043-0.163$, allowing us to obtain spatially resolved optical spectroscopy with the Keck ESI $1^{\prime \prime} \times 20^{\prime \prime}$ long slit. The data were reduced as described in Martin (2005), yielding spectral resolution of $\sim 70 \mathrm{~km} \mathrm{~s}^{-1}$ and spatial resolution $\approx 0$ '. 8 limited by the atmospheric seeing. The median spatial resolution is $1.5 \mathrm{kpc}$, with a range of $0.7-2.1 \mathrm{kpc}$ depending on redshift.

\section{RESULTS}

In Paper I, we decomposed the emission-line profiles into multiple Gaussian components. An example of this decomposition is shown in Figure 1. Because we jointly fit several transitions (H $\alpha,[\mathrm{N}$ II] $\lambda \lambda 6548,6583$, [O I] $\lambda 6300,[\mathrm{~S} \mathrm{II}] \lambda \lambda 6716$, $6730, \mathrm{H} \beta$, [O III] $\lambda 5007$ ), we measured diagnostic line ratios for each velocity component. Using kpc-scale apertures, we mapped the Doppler shift, velocity width, and line fluxes across each galaxy. The results, provided in Table 3 of Paper I, were used to classify the excitation mechanism of each component as $\mathrm{H}$ II-like or shock-like. Here, we explore the relationship between the gas kinematics and the excitation mechanism.

For each velocity component at a given spatial location, we classified the excitation mechanism as $\mathrm{H}$ II-like or shock-like using diagnostic ratios of line fluxes (Kewley et al. 2006). The $[\mathrm{O}$ I] $/ \mathrm{H} \alpha$ ratio plays a major role in this categorization due to its high sensitivity to shocks. In cases where $[\mathrm{O} \mathrm{I}] / \mathrm{H} \alpha$ is obscured by telluric absorption, we rely on $\left[\mathrm{S}{ }_{\mathrm{II}}\right] / \mathrm{H} \alpha$ and $[\mathrm{N} \mathrm{II}] / \mathrm{H} \alpha$ to make this distinction. If the errors in the flux ratio cross the maximum star formation line, then the excitation mechanism is designated "unclear." We note that our "unclear" category differs from the composite classification of Kewley et al. (2006), defined by line ratios between the empirically defined maximum star formation limit (Kauffmann et al. 2003) and the maximum star formation (Kewley et al. 2001). We further describe these categories and the fitting method in Paper I.

Figure 2 shows a histogram of the velocity dispersions of the components from all apertures across all galaxies divided by excitation class. Spectral components classified as " $\mathrm{H} \mathrm{II-}$ like" are narrow in linewidth, having $\sigma_{v} \leqslant 150 \mathrm{~km} \mathrm{~s}^{-1}$. The components identified as "shock-like," however, present a highvelocity tail at $\sigma_{v}>150 \mathrm{~km} \mathrm{~s}^{-1}$. Clearly the broad components are preferentially shock excited.

We show the Doppler shift of all the components in Figure 3. The narrow, shock-like components (and the H II-like 


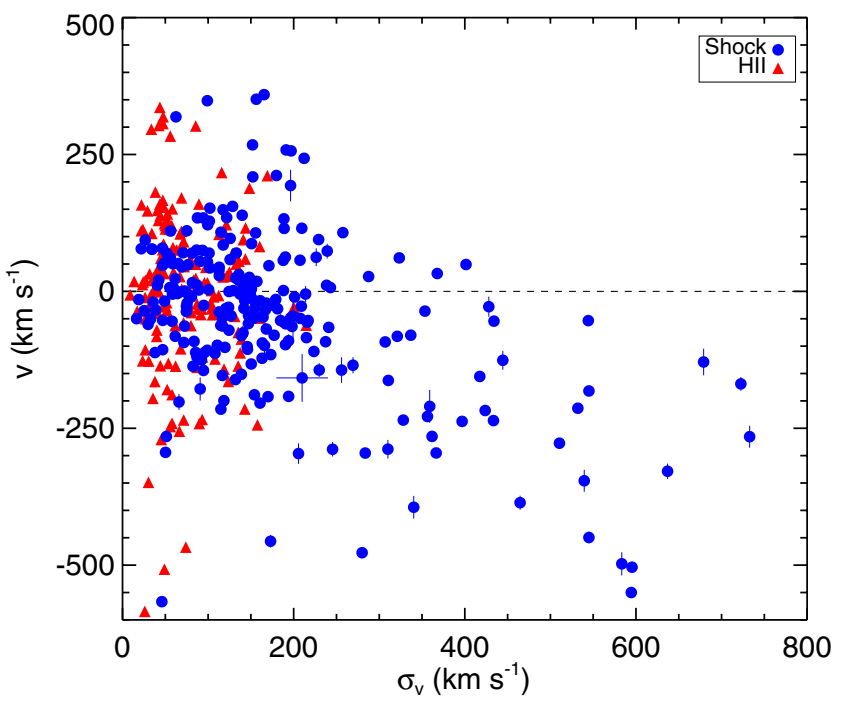

Figure 3. We find that the components identified as shock-like with $\sigma_{v}<$ $150 \mathrm{~km} \mathrm{~s}^{-1}$ have a similar distribution in Doppler shift as the $\mathrm{H}$ II components. The shock-like components with $\sigma_{v}>150 \mathrm{~km} \mathrm{~s}^{-1}$, however, present significant blueshifts.

(A color version of this figure is available in the online journal.)

components) are detected over distances of 15-30 kpc along the slit. The velocity offsets relative to the galaxy redshift are positive in one direction and negative on the opposite side of the nucleus. Figure 5 of Paper I demonstrates that the position-velocity diagrams for these narrow, shock-like components are typically consistent with the projection of a galactic rotation curve, suggesting that the process producing narrow, shock-like emission components is associated with a gas disk.

The broader components with $\sigma_{v}>150 \mathrm{~km} \mathrm{~s}^{-1}$, however, do not show rotation gradients, suggesting that a different physical mechanism is involved in producing the broad emission. Figure 3 shows that the broad, shocked components are nearly always blueshifted, reaching a maximum of $500 \mathrm{~km} \mathrm{~s}^{-1}$.

Because ULIRGs are very dusty galaxies, their nuclei are not transparent at optical wavelengths. Hence, the emissionline radiation that we detect at the center of ULIRGs must be emitted on the near side of the dusty, gaseous disk. We therefore conclude that the blueshifted emission comes from outflowing gas on the near side of the galaxy rather than infall on the far side. The broad emission in adjacent apertures maintains similar kinematics, which suggests that these regions have similar dust obscuration. In support of this outflow interpretation, we note that the broad, shocked emission spans a velocity range quite similar to that of the blueshifted $\mathrm{Na}$ I absorption troughs previously identified in these spectra (Martin 2005, 2006).

Using the spatial information along the slit, we traced the extent of each spectral component in Paper I. Figure 4 shows how the spatial extent of an emission component varies with its velocity dispersion and excitation classification. Broad, shocked emission in ULIRGs is usually spatially extended but is not detected as far away from the nucleus as is the narrow component. The broad, shocked component can typically be traced to $\sim 5 \mathrm{kpc}$ away from the peak continuum emission and up to $6 \mathrm{kpc}$ in IRAS $03158+4227$. We discuss the source of these broad, shocked outflows in Section 4.

\section{OUTFLOW SOURCE}

In general, the broad emission components are blueshifted to high velocity relative to the systemic velocity, and only

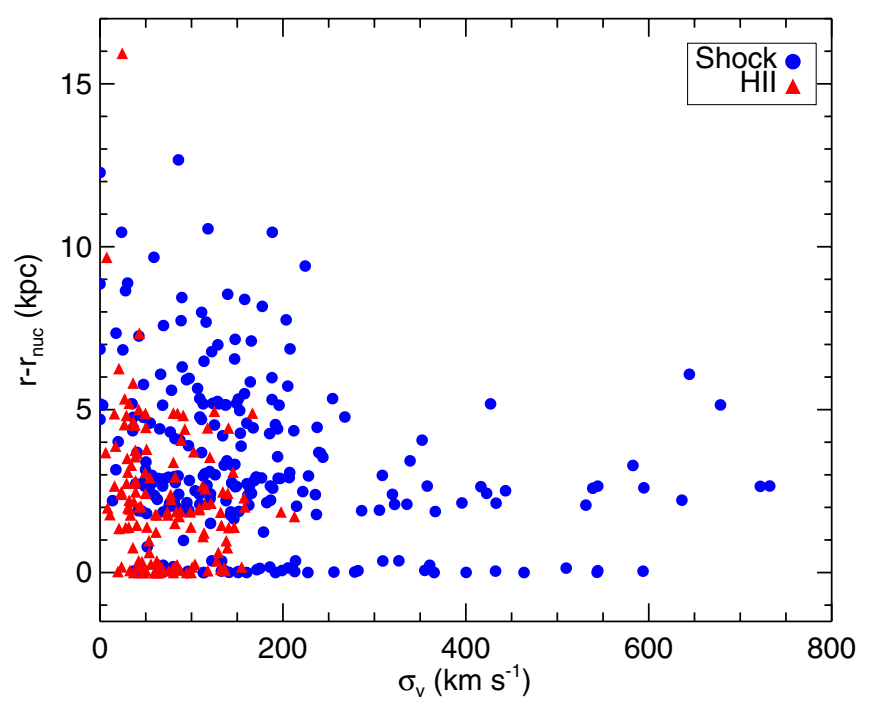

Figure 4. $\mathrm{H}$ II- and shock-like components with $\sigma_{v}<150 \mathrm{~km} \mathrm{~s}^{-1}$ extend to regions $15 \mathrm{kpc}$ away from their associated nuclei. Components with $\sigma_{v}>150 \mathrm{~km} \mathrm{~s}^{-1}$, however, remain within $5 \mathrm{kpc}$ of the nucleus. The gap between the points at $r=r_{\text {nuc }}$ and the rest of the distribution reflects the spatial resolution of the spectroscopy.

(A color version of this figure is available in the online journal.)

a few components are redshifted as shown in Figure 3. For emission, a blueshifted line profile can be interpreted in two ways - outflow on the near side of the galaxy or inflow on the far side of the galaxy. In the case of dusty ULIRGs, however, the presence of dust obscuration allows a unique identification of the blueshift as outflow; photons coming from infalling gas on the far side of the galaxy would have a longer path length through dusty regions, thereby increasing the amount of extinction. The outflow interpretation is further supported by the spatial extent of the blueshifted lines identified in Figure 4. The spatial extent of broad blueshifted emission suggests that the emission is associated with regions beyond the deepest part of the gravitational potential well. In the following sections, we explore the possible physical origins of the outflow.

\subsection{Star Formation versus AGNs as a Power Source}

The power source of ULIRGs has been under debate for more than 20 years, owing to the obscuration in optical and shorter wavelengths by dust. Using the Spitzer Infrared Spectrograph, Veilleux et al. (2009) previously examined the power source of 17 of the 39 ULIRGs included in the Paper I sample. Indicators of the strength of AGN contributions come from ratios of mid-infrared emission lines [O IV]/[Ne II] and [Ne v]/[Ne II] (Armus et al. 2004, 2006, 2007; Veilleux et al. 2009); however, these ratios are well constrained for only 3 of the 17 ULIRGs in this sample: two of the objects (IRAS 01572+0009, IRAS 12072-0444) show 90\% AGN contributions to $L_{\mathrm{bol}}$, while the third (IRAS $15001+1433$ ) that is well constrained shows an $\sim 50 \%$ AGN contribution. Measurements of the mid-IR line ratios for the other $14 \mathrm{ULIRGs}^{3}$ place a $\lesssim 30 \%$ upper limit on the AGN contribution to their bolometric luminosity. Therefore, AGNs do not appear to be the dominant power source in our ULIRG sample.

\footnotetext{
3 The remaining 14 ULIRGs in the Veilleux et al. (2009) study are IRAS 00188-0856, IRAS 01003-2237, IRAS 01298-0744, IRAS $03521+0028$, IRAS $10378+1108$, IRAS $10565+2248$, IRAS 11095-0238, IRAS 11506+1331, IRAS 13451+1232, IRAS 15130-1958, IRAS 15462-0450, IRAS 16090-0139, IRAS 17208-0014, and IRAS 20414-1651.
} 


\subsubsection{AGN Broad-line Region}

Although the AGN is generally not the dominant source of luminosity in these ULIRGs, we still need to investigate whether the broad lines could be directly related to the AGN. A broadline region (BLR) is a common feature in Seyfert 1 and 1.5 galaxies, appearing as permitted and intercombination emission lines broadened to 1000-25,000 $\mathrm{km} \mathrm{s}^{-1}$ FWHM (Osterbrock $\&$ Ferland 2006). The weakness of forbidden transitions in the BLR is explained by the high electron density, $n_{e}>$ $10^{9} \mathrm{~cm}^{-3}$, which enhances collisional de-excitation (Peterson 2006). Reverberation mapping places the BLR size at much less than 1 pc (Bentz et al. 2009; Brewer et al. 2011), suggesting fast-moving gas in the deep gravitational potential well of a supermassive black hole (Dietrich et al. 1999). In our ULIRG spectra, emission from an AGN BLR would be confined to the central aperture $\left(\sim 1^{\prime \prime}\right)$.

The broad emission lines we observe in our ULIRG spectra, however, extend beyond a spatial resolution element, calling into question the BLR as a source. Additionally the broad emission features appear not only in the permitted transitions but also in the forbidden transitions. These observations, along with the low electron density measurements $\lesssim 100 \mathrm{~cm}^{-3}$ from [S II] line ratios, rule out an AGN BLR as the source of the broad, shocked emission components.

\subsubsection{Narrow-line Region}

In the emission-line ratio diagrams, we use the line defining the maximum excitation via extreme star formation (Kewley et al. 2006) to distinguish $\mathrm{H}$ II-like emission from shock-like emission. Above this line, the emission-line ratios are typically attributed to fast shocks in the NLRs of Seyfert galaxies or LINERs, where the gas density ahead of the shock determines the influence of the radiative precursor on the overall emissionline ratio. Gas-rich Seyfert galaxies have a larger contribution from the radiative precursor, resulting in larger [O III] $/ \mathrm{H} \beta$ ratios. LINERs are suspected to have lower gas densities ahead of the shock, which leads to lower $[\mathrm{O} \mathrm{III}] / \mathrm{H} \beta$ ratios (Dopita \& Sutherland 1995).

To determine whether the shock-like emission we observe is coming from the NLRs of an AGN, we estimate the expected size of this region. The size of a shocked AGN NLR region is empirically related to the luminosity of the $[\mathrm{O}$ III] $\lambda 5007$ emission (Bennert et al. 2002; Greene et al. 2011). For each galaxy, we estimate $L_{[\mathrm{O} \text { III] }}$ from the broad, shock-like components and predict the expected size of an AGN NLR. We use the scaling relation developed by Greene et al. (2011), which does not include corrections for in situ dust extinction. The [O III] luminosities $\left(L_{\left[\mathrm{O}_{\mathrm{III}}\right]}\right)$ used in this scaling relation only include spectral components where $\log ([\mathrm{O} \mathrm{III}] / \mathrm{H} \beta)$ is greater than 0.5 to avoid flux contributions from an $\mathrm{H}$ II region. Since either nucleus in a merging pair can host an AGN, we measure the luminosity for each galaxy in the sample-increasing the total galaxy count in this study to 48 . We restrict our analysis to spectral components where $\log ([\mathrm{O} \mathrm{III}] / \mathrm{H} \beta)>0.5$ to avoid flux contributions from $\mathrm{H}$ II regions. The range of luminosities within these galaxies spans several orders of magnitude $\left(1.9 \times 10^{37}<L_{[\mathrm{O} \text { III }]}<\right.$

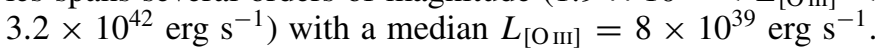
Slit losses create a factor of $\sim 2$ systematic error in the measurement of $L_{[\mathrm{O} I \mathrm{II}]}$; however, the scaling relation is not strongly influenced by this factor. For the size measurement, we estimate the error as $1 / 2$ the size of the average aperture $(\sim 0$ '. 4$)$. In nine galaxies (in seven ULIRG systems-IRAS 00188-0586,
IRAS 01003-2238, IRAS 05246+0103 (both nuclei), IRAS 08311-2459, IRAS 10378+1109, IRAS 13451+1232 (both nuclei), and IRAS 15130-1958), we find that the radius predicted by the scaling relation (Greene et al. 2011) exceeds or is consistent with the size along the slit.

We compare our AGN identification method to studies that have identified AGN-driven outflow via the IR emission lines [Ne III] and [Ne v] (Spoon \& Holt 2009). Three of the five ULIRGs in the overlapping sample have AGNs identified by our $L_{[\mathrm{O} \text { III }}$ scale size versus outflow size method. Our method has not identified IRAS $15462-0405$, in which the kinematics of [O III] varied differently from the other emission lines. This variation in kinematics may also be a basis for identifying AGN contributors to the outflow. In IRAS 12071-0444, the heavy extinction evidenced by the masking of the broad component in $\mathrm{H} \beta$ is a possible contributor to the misidentification.

In another study (Westmoquette et al. 2012), the ULIRGs IRAS 09111-1007, IRAS 17208-0014, IRAS 19297-0406, IRAS 20046-0623, and IRAS 20414-1651 are examined using the VIMOS integral field unit. In two of these ULIRGs (IRAS 09111-1007 and IRAS 19297-0406), the study detects outflows using measurements of the $\mathrm{H} \alpha$, [N II], [S II], and [O I], with similar kinematics to those found by this study. Their interpretation describes $\mathrm{AGN}$ as the mechanism that creates the features; however, using the AGN scaling relation with $L_{\left[\mathrm{O}_{\text {III }}\right]}$ and the size of the emission region, we find that the star formation is more likely responsible. Both studies agree on the three remaining galaxies in our overlapping samples, which do not show evidence for outflow and are starburst dominated.

The majority of galaxies have $L_{[\mathrm{O} \text { III }}$ and spatial distributions that do not suggest excitation via AGNs. In the 11 galaxies (nine $\left.\mathrm{ULIRGs}^{4}\right)$, no components appear above the $\log ([\mathrm{O} \mathrm{III}] / \mathrm{H} \beta)>$ 0.5 cutoff, but we still detect shock-like line ratios up to $6 \mathrm{kpc}$ from the closest nucleus, suggesting that some other mechanism is responsible for the ionization. For 14 galaxies (11 ULIRGs $\left.{ }^{5}\right)$, components with strong $\log ([\mathrm{O} \mathrm{III}] / \mathrm{H} \beta)$ appear only in regions outside the nuclear aperture. Galaxies that have a strong extended AGN presence would more likely consist of a contiguous region that includes the nucleus, suggesting that these are also not part of an NLR. Excluding the nine galaxies identified as AGNs, the remaining 14 galaxies have regions with shocked gas at radii greater than the estimated NLR size. In general, most cases do not favor AGNs as the source of the observed emission line profile and instead are consistent with shocks driven by stellar winds and supernovae, which motivates us to consider whether this alternative is energetically feasible.

\subsection{Energetics of Supernova Feedback}

Excluding the AGN-dominanted cases IRAS 01572+0009 and IRAS 12072-0444, the SFRs in these ULIRGs indicate that an enormous amount of mechanical power is deposited in the interstellar medium (ISM) by massive stars. In this section, we compare the estimated mechanical power to the power in the bulk outflow of warm ionized gas. We stress that our estimates of the mass and kinetic energy in the bulk flow are factor of

\footnotetext{
4 Galaxies without components with [O III] $/ \mathrm{H} \beta>0.5$ : IRAS 00153+5454 (both nuclei), IRAS 11095-0238, IRAS 15462-0405, IRAS 16474+3430, IRAS 17028+5817, IRAS 17208-0014, IRAS 18470+3233 (both nuclei), IRAS $19458+0944$, and IRAS $23365+3604$.

5 Galaxies with $[\mathrm{O} \mathrm{III}] / \mathrm{H} \beta>0.5$ only outside the nucleus: IRAS 03158+4227, IRAS 08030+5243, IRAS 10494+4424, IRAS $10565+2448$, IRAS $11506+1331$ (both nuclei), IRAS $15245+1019$ (both nuclei), IRAS 16090-0139, IRAS 16487+5447 (both nuclei), IRAS 17574+0629, IRAS 20046-0623, and IRAS 23327+2913.
} 
\% accuracy. Uncertainty about the filling factor of the warm ionized gas and the large-scale geometry of the outflow dominate the systematic error. The mass, energy, and momentum carried by the outflowing, warm ionized gas should clearly be viewed as a lower limit on the total amounts carried by all phases of the wind, which likely includes substantial components of both coronal gas and molecular gas.

\subsubsection{Mass of Warm Ionized Gas}

For each ULIRG that shows broad, shock-like emission components, we estimate the outflowing mass of warm ionized gas via the product of gas density and the volume occupied by the warm ionized phase. Critical to these estimates is the filling factor, which represents the substructure of the emitting gas. Using Case B recombination theory, we relate the measured luminosities, surface brightnesses, and volumes to the outflowing mass.

The fraction of the total volume $V$ filled with warm ionized gas is called the filling factor. For $\mathrm{H}$ II regions, this filling factor,

$$
f \equiv \frac{\left\langle n_{e}^{2}\right\rangle}{n_{e}^{2}},
$$

is computed from the rms electron density obtained from the $\mathrm{H} \alpha$ luminosity or surface brightness and the electron density computed from a density-sensitive doublet ratio such as [S II] $\lambda \lambda 6717,31$. Values of the filling factor range from $0.001<$ $f<0.1$ in nearby H II regions (Searle 1971; Kennicutt 1984; Kaufman et al. 1987), which can have a very significant impact on the estimated mass. The typical filling factor may be different in the ULIRGs, given the difference in SFR from these $\mathrm{H}$ II regions in normal galaxies, but these values provide a point of comparison. In the ULIRG spectra, the flux ratio for the [S II] doublet is typically consistent with the low-density limit, indicating $n_{e} \lesssim 100 \mathrm{~cm}^{-3}$, except in the central apertures of IRAS 01003-2238, IRAS 05246+0103, IRAS 08311-2459, IRAS 09583+4714, and IRAS 23327+2913, where $n_{e} \approx$ $500 \mathrm{~cm}^{-3}$. For our mass estimates, we adopt a fiducial value of $n_{e}=100 \mathrm{~cm}^{-3}$ but carry along the scaling with density explicitly in our results.

The outflow mass is

$$
M_{\mathrm{out}}=\mu m_{\mathrm{H}} V n_{e} f
$$

where $n_{e}$ is the electron density, $m_{\mathrm{H}}$ is the mass of hydrogen, and the mass per $\mathrm{H}$ atom is $\mu=1.4 \mathrm{amu}$ when helium is included. The factor $V n_{e} f$ in Equation (2) is simply related to the $\mathrm{H} \alpha$ luminosity by

$$
L_{\mathrm{H} \alpha}=\gamma^{\prime}\left\langle n_{e}^{2}\right\rangle V=\gamma^{\prime} n_{e}^{2} f V,
$$

where $\gamma^{\prime}$ is the effective volume emissivity. For this estimate based on luminosity, we substitute $M_{\mathrm{L}}$ for $M_{\text {out }}$. By eliminating the common factors between Equations (2) and (3), the outflowing mass,

$$
M_{\mathrm{L}}=\frac{\mu m_{\mathrm{H}} L_{\mathrm{H} \alpha}}{\gamma^{\prime} n_{e}},
$$

can be estimated from the luminosity of the broad, shocked emission component for any assumed gas density, $n_{e}$.

The effective volume emissivity $\left(\gamma^{\prime}\right)$ varies depending on the temperature and excitation mechanism. For photoionized regions with $T=10^{4} \mathrm{~K}, \gamma^{\prime}=\gamma_{\text {phot }}=\alpha_{\mathrm{H} \alpha}^{\text {eff }} h v=3.56 \times$ $10^{-25} \mathrm{erg} \mathrm{cm}^{3} \mathrm{~s}^{-1}$ in Case B recombination theory (Osterbrock

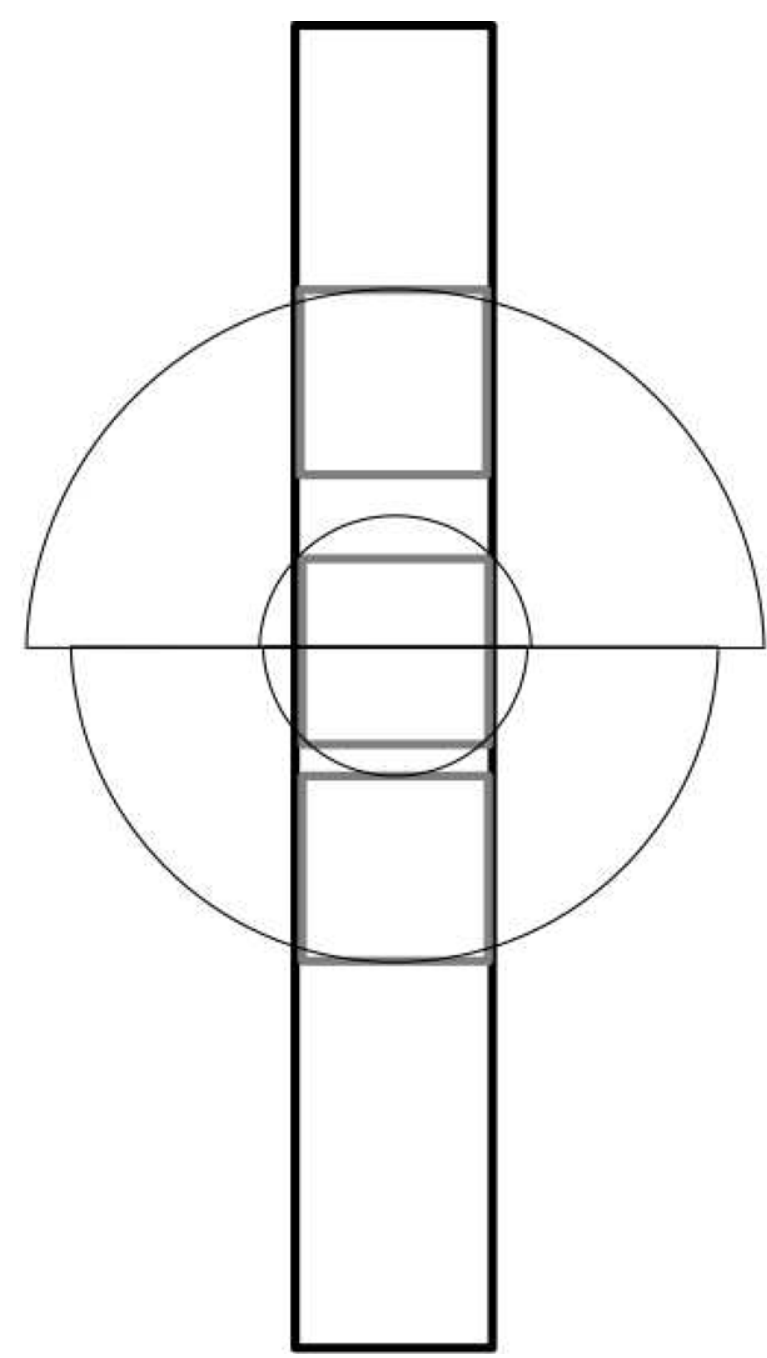

Figure 5. Long black rectangle represents the slit, the gray boxes represent apertures along the slit, and the annuli are the total estimated regions for each position. The number of outflows detected in ULIRGs (75\%-80\%; Rupke et al. 2002; Martin 2005) suggests that a biconical outflow cone has a large opening angle $\sim 145^{\circ}$. This allows us to use a sphere as a rough approximation to the geometry. We estimate the luminosity contributed by regions outside the slit by assuming circular symmetry with an equal surface brightness in the half annulus to that of the measured aperture. The central aperture coincides with the position of the $r$-band continuum peaks along the slit.

\& Ferland 2006). In shocked regions, collisional excitation and ionization can make $\gamma^{\prime}$ larger or smaller than $\gamma_{\text {collision }}$ by a factor of two to three (Goerdt et al. 2010; Genzel et al. 2011). Since the photoionization case is in the middle of the plausible range

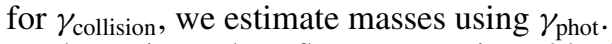

The estimated outflow masses in Table 1 range from $1.8 \times$ $10^{6} M_{\odot}$ to $3.9 \times 10^{8} M_{\odot}$, with median and standard deviations $40 \times 10^{6} M_{\odot}$ and $84 \times 10^{6} M_{\odot}$, respectively. Systematic errors in these mass estimates are difficult to quantify. For example, Figure 5 illustrates our estimate of aperture correction to the observed flux. In addition, corrections for internal $\mathrm{H} \alpha$ extinction can be large. The large outflow mass in IRAS 20087-0308, for example, is due in large part to the extinction correction. For individual galaxies, the masses in Column 7 of Table 1 should be interpreted as rough estimates accurate to a factor of 2-3.

Measurements of $\mathrm{H} \alpha$ surface brightness provide a sanity check on the assumed electron density or equivalently $\left\langle n_{e}^{2}\right\rangle^{1 / 2} f^{-1 / 2}$ by Equation (1). The $\mathrm{H} \alpha$ surface brightness 
Table 1

Outflow Parameters

\begin{tabular}{|c|c|c|c|c|c|c|c|c|c|c|c|c|}
\hline $\begin{array}{l}\text { IRAS Name } \\
\text { (1) }\end{array}$ & $\begin{array}{l}L_{\mathrm{IR}} \\
(2)\end{array}$ & $\begin{array}{l}v_{\text {out }} \\
(3)\end{array}$ & $\begin{array}{c}r_{\text {out }} \\
\text { (4) }\end{array}$ & $\begin{array}{l}t_{\text {out }} \\
(5)\end{array}$ & $\begin{array}{c}L_{\text {tot }} \\
(6)\end{array}$ & $\begin{array}{r}M_{\mathrm{L}} \\
(7)\end{array}$ & $\begin{array}{l}M_{\Sigma} \\
(8)\end{array}$ & $\begin{array}{r}\dot{M}_{\mathrm{L}} \\
(9)\end{array}$ & $\begin{array}{l}\dot{M}_{\Sigma} \\
(10)\end{array}$ & $\begin{array}{l}\text { SFR } \\
(11)\end{array}$ & $\begin{array}{c}\eta_{\mathrm{L}} \\
(12)\end{array}$ & $\begin{array}{c}\eta_{\Sigma} \\
(13)\end{array}$ \\
\hline IRAS $00153+5454$ & 12.10 & 270 & 3.71 & 13.31 & 51.5 & 17 & 47 & 1.3 & 3.6 & 68 & 0.02 & 0.05 \\
\hline IRAS $00188-0856^{\mathrm{a}}$ & 12.33 & 660 & 1.57 & 2.32 & 407. & 130 & 31 & 58 & 13 & 128 & 0.45 & 0.10 \\
\hline IRAS $01003-2238^{a}$ & 12.25 & 1140 & 3.66 & 3.13 & 203. & 67 & 155 & 21 & 50 & 96 & 0.22 & 0.52 \\
\hline IRAS 01298-0744 & 12.29 & 240 & 11.27 & 44.98 & 86.6 & 28 & 620 & 0.6 & 14 & 57 & 0.01 & 0.24 \\
\hline IRAS $03158+4227$ & 12.55 & 1330 & 7.22 & 5.30 & 181. & 59 & 423 & 11 & 80 & 191 & 0.06 & 0.42 \\
\hline IRAS $05246+0103^{a}$ & 12.05 & 850 & 3.10 & 3.56 & 520. & 170 & 110 & 48 & 31 & 60 & 0.80 & 0.52 \\
\hline IRAS $08311-2459^{a}$ & 12.40 & 600 & 4.94 & 8.08 & 338. & 110 & 320 & 14 & 40 & 135 & 0.10 & 0.30 \\
\hline IRAS 09111-1007 & 11.98 & 290 & 1.38 & 4.68 & 26.3 & 8 & 7.3 & 1.8 & 1.6 & 51 & 0.04 & 0.03 \\
\hline IRAS $09583+4714$ & 11.98 & 350 & 4.42 & 12.27 & 413. & 140 & 320 & 11 & 26 & 51 & 0.20 & 0.50 \\
\hline IRAS $10378+1109^{a}$ & 12.23 & 1000 & 3.80 & 3.72 & 267. & 88 & 150 & 24 & 41 & 92 & 0.26 & 0.45 \\
\hline IRAS $10494+4424$ & 12.15 & 730 & 4.34 & 5.78 & 80.2 & 26 & 88 & 4.6 & 15 & 76 & 0.06 & 0.20 \\
\hline IRAS $10565+2448$ & 11.98 & 250 & 2.66 & 10.54 & 23.2 & 8 & 29 & 0.7 & 2.8 & 66 & 0.01 & 0.04 \\
\hline IRAS $12071-0444$ & 12.31 & 570 & 7.95 & 13.57 & 15.9 & 5 & 210 & 0.4 & 15 & 25 & 0.02 & 0.61 \\
\hline IRAS $15130-1958^{a}$ & 12.03 & 890 & 3.65 & 4.03 & 29.5 & 10 & 51 & 2.4 & 13 & 24 & 0.10 & 0.53 \\
\hline IRAS 16090-0139 & 12.48 & 640 & 6.61 & 10.07 & 17.0 & 6 & 86 & 0.6 & 8.5 & 179 & 0.003 & 0.05 \\
\hline IRAS $16487+5447$ & 12.12 & 400 & 5.79 & 14.01 & 147. & 49 & 360 & 3.5 & 26 & 71 & 0.05 & 0.36 \\
\hline IRAS $17028+5817$ & 12.11 & 200 & 1.33 & 6.42 & 121. & 40 & 14 & 6.2 & 2.1 & 69 & 0.09 & 0.03 \\
\hline IRAS $18368+3549$ & 12.19 & 370 & 4.09 & 10.70 & 333. & 110 & 140 & 10. & 13 & 83 & 0.12 & 0.16 \\
\hline IRAS 19297-0406 & 12.36 & 730 & 4.30 & 5.74 & 233. & 77 & 180 & 13 & 31 & 123 & 0.11 & 0.25 \\
\hline IRAS 20087-0308 & 12.39 & 330 & 5.62 & 16.48 & 1170 & 390 & 760 & 24 & 46 & 132 & 0.18 & 0.35 \\
\hline IRAS $23327+2913$ & 12.03 & 630 & 3.81 & 5.95 & 60.5 & 20 & 99 & 3.4 & 17 & 58 & 0.06 & 0.29 \\
\hline IRAS $23365+3604$ & 12.13 & 580 & 0.89 & 1.50 & 5.52 & 2 & 1.6 & 1.2 & 1.1 & 73 & 0.02 & 0.02 \\
\hline
\end{tabular}

Notes. The objects in this table are restricted to those that have at least one aperture with a shock-like spectral component with $\sigma_{v}>150 \mathrm{~km} \mathrm{~s}{ }^{-1}$. Column 1: IRAS Name. Column 2: total infrared luminosity $\log \left(L_{\mathrm{IR}} / L_{\odot}\right)$ (Murphy et al. 1996). Column 3: outflow velocity taken as $v_{\text {out }}=|v|+\sigma_{v}$ $\left(\mathrm{km} \mathrm{s}^{-1}\right)$ from Paper I (Table 3). Since we are unsure of the degree to which projection effects play a role in the measured velocity, we take the maximum $v_{\text {out }}$ of the apertures. Column 4: maximum radius of outflow $(\mathrm{kpc})$. $r_{\text {out }}$ is defined by the maximum separation between an aperture that shows outflow and the continuum peak. Column 5: outflow timescale (Myr), calculated from $r_{\text {out }} / v_{\text {out }}$. Column 6: total luminosity of H $\alpha$ from the outflow components $\left(L_{\text {tot }} / 10^{40} \mathrm{erg} \mathrm{s}^{-1}\right)$. We correct the $\mathrm{H} \alpha$ emission for Galactic dust extinction using the reddening curve from Cardelli et al. (1989) and values of $E(B-V)$ and $A_{V}$ from the Infrared Science Archive maps (Schlegel et al. 1998). We use the measured Balmer decrement and the Calzetti et al. (1994) reddening curve to correct for internal extinction. Column 7: mass of outflowing gas in units of $10^{6} M_{\odot}$ from $L_{\mathrm{tot}}$ and Equation (4) assuming $n_{e}=100 \mathrm{~cm}{ }^{-3}$ and $\gamma^{\prime}=\gamma_{\text {phot }} . M_{\mathrm{L}}=3.3 \times 10^{5} M_{\odot}\left(100 \mathrm{~cm}^{-3} / n_{e}\right)\left(L_{\mathrm{H} \alpha} / 10^{40} \mathrm{erg} \mathrm{s}^{-1}\right)$. Column 8: mass of outflowing gas in units of $10^{6} M_{\odot}$ from $\Sigma$. $\Sigma$ is calculated per aperture using the measured luminosity and area of each aperture. The mass comes from Equation (6) with an assumed hemisphere geometry, where the maximum path length through the sphere is defined by the radius $\delta s=r_{\text {out }}$ and $f=0.01$. Within each aperture the path length through the sphere varies as $s=\left(r_{\text {out }}^{2}-\left(r_{\text {aperture }}-r_{\text {nucleus }}\right)^{2}\right)^{1 / 2} . M_{\Sigma}=2.42 \times 10^{6} M_{\odot} f^{1 / 2}\left(A / 1 \mathrm{kpc}^{2}\right)(s / 1 \mathrm{kpc})^{1 / 2}\left(\Sigma / 10^{-15} \mathrm{erg} \mathrm{s}^{-1} \mathrm{arcsec}^{-2}\right)^{1 / 2}$. Column 9 : outflow rate in units of $M_{\odot} \mathrm{yr}^{-1}$ estimated from $L_{\text {tot }}$ described in Section 4.2.2. Column 10: outflow rate in units of $M_{\odot} \mathrm{yr}^{-1}$ estimated from $\Sigma$ with an assumed hemisphere geometry. Column 11: star formation rate in units of $M_{\odot} \mathrm{yr}^{-1}$ estimated from $L_{\mathrm{IR}}$ (Murphy et al. 1996) and relation SFR $=$ $L_{\mathrm{IR}} / 13.0 \times 10^{9} L_{\odot}$. This relation uses the Kennicutt (1998) relation adjusted from a stellar mass range of $0.1-100 M_{\odot}$ to a stellar mass range from 1 to $100 M_{\odot}$. This star formation rate is also corrected for AGN contribution to $L_{\mathrm{bol}}$ for the objects included in Veilleux et al. (2009). In cases where upper limits to the AGN fraction are not provided for an individual object in Veilleux et al. (2009), we use the average AGN contribution of 30\%. Column 12: efficiency of outflow from $\dot{M}_{\mathrm{L}}$. Column 13: efficiency of outflow from $\dot{M}_{\Sigma}$ with an assumed hemisphere geometry and filling factor $f=0.01$.

a Possible AGN-driven source.

$\Sigma=L_{\mathrm{Ha}} / A$ depends on the three-dimensional shape of the outflow since the volume is the product of the projected area $A$ and the depth of the emitting region, $\Delta s$, along the sightline. The surface brightness depends on density as

$$
\Sigma=\left\langle n_{e}^{2}\right\rangle \gamma^{\prime} \Delta s
$$

By analogy to Equation (4), the inferred outflow mass is

$$
M_{\mathrm{out}}=M_{\Sigma}=\left(\frac{\mu m_{\mathrm{H}} A \Sigma}{\gamma^{\prime} n_{e}}\right)=\mu m_{\mathrm{H}} L\left(\frac{\Delta s f}{\gamma^{\prime} \Sigma}\right)^{\frac{1}{2}}
$$

In Table 1 , Column 8 provides examples of the outflow mass estimates obtained by this method.

If our density estimate $n_{e}$ is reasonable, then equating $M_{\Sigma}$ and $M_{L}$ should imply reasonable values for the product of the filling factor and the depth of the emitting region along the line of sight. If we adopt $f=0.01$, then the required path length through the emitting region is often only a few hundred pc. Such a small path length would arise only if the emitting region had a hollow shell structure. If the emitting region is roughly spherical, then $\Delta s \approx \sqrt{A}$, and the implied filling factor is often small, $f \sim 10^{-3}$. The real values of $s$ and $f$ that unify the mass estimates will depend on each individual case. The bottom line is that the volume filling factor of the warm ionized gas may be tiny, thereby leaving lots of volume to be filled by gas at a different temperature.

\subsubsection{Outflow Velocity and Timescale}

Estimates of the outflow timescale follow from the geometry along the slit and the kinematics measured from the emissionline profile. In Table 1 , we define the outflow velocity $\left(v_{\text {out }}\right)$ as $|v|+\sigma_{v}\left(\mathrm{~km} \mathrm{~s}^{-1}\right)$ taken from Table 3 in Paper I. The median $v_{\text {out }}$ in this sample is $580 \mathrm{~km} \mathrm{~s}^{-1}$, with values that range from 200 to $1330 \mathrm{~km} \mathrm{~s}^{-1}$. 
When we estimate the outflow timescale using the outflow radius and velocity, $t_{\text {out }}=r_{\text {out }} / v_{\text {out }}$, the implied mass loss rate of warm ionized gas is $\dot{M}=M_{\text {out }} / t_{\text {out }}$. For the estimate of mass outflow using luminosity $\left(M_{\mathrm{L}}\right)$, we find a range of $\dot{M}$ from 0.4 to $58 M_{\odot} \mathrm{yr}^{-1}$ where the median is $4.6 M_{\odot} \mathrm{yr}^{-1}$. The largest value of $\dot{M}$ is in IRAS $00188-0856$, due mostly to the moderate $v_{\text {out }}$ and small $r_{\text {out }}$. The mass estimates using surface brightness and a spherical geometry $\left(M_{\Sigma}\right)$ give larger outflow rates that range from 1 to $80 M_{\odot} \mathrm{yr}^{-1}$ where the median is $15.2 M_{\odot} \mathrm{yr}^{-1}$. In this case, the largest outflow rate is in IRAS $03158+4227$, which has a large $v_{\text {out }}$ and $r_{\text {out }}$, but in the surface brightness case, the large $r_{\text {out }}$ has a greater influence.

\subsection{3. $\dot{M}$ versus $S F R$}

Galactic wind models require estimates of star formation's efficiency $(\eta)$ in removing gas from the star-forming region. This parameter informs the timescale over which a galaxy will form stars before the stellar population itself removes gas and shuts down star formation. We estimate $\eta$ as the ratio of mass outflow rate to the SFR measured from IR luminosities in Murphy et al. (1996) (Table 1). The SFR estimates use the relation SFR = $L_{\mathrm{IR}} / 13.0 \times 10^{9} L_{\odot}($ Kennicutt 1998), adjusted for a stellar mass range from 1 to $100 M_{\odot}$ and correcting the $L_{\mathrm{IR}}$ for the AGN fraction. While there are few AGN-driven outflow sources in this sample, we find that $\eta$ for these are distributed up to $\eta=0.8$. In contrast, there are more star-formation-driven outflows, and they are concentrated below $\eta=0.25$.

In this sample of galaxies, the outflow rates typically yield $\eta \sim 10^{-1}$ but span the range $10^{-3}<\eta<1$. We emphasize, however, that this measurement refers only to the warm ionized gas, which may not be the dominant phase of the outflowing gas. The mass outflow rates in the cool gas traced by $\mathrm{Na}$ I are at least a few tenths of the SFR $(\eta \equiv \dot{M} /$ SFR $\approx 0.1)$ but are poorly constrained due to the large variations in ionization parameter among ULIRGs (Martin 2005; Murray et al. 2007). Hot winds and molecular outflows may also carry significant mass.

\subsubsection{Shock Energetics}

The presence of outflows implies the injection of mechanical energy into the gas. Stellar wind bubbles (Weaver et al. 1977; Shull \& McKee 1979) provide the basis for the simplest model of the feedback response of the interstellar gas. In these models, shocks direct $55 \%$ of the feedback energy into thermal energy of the low-density, coronal bubble and the other $45 \%$ into an expanding shell of swept-up ISM. Approximately $60 \%$ of the shell energy is radiated away in this model, suggesting that luminosity of the shocks would be $27 \%$ of the feedback power and that the kinetic energy of the shell would correspond to $20 \%$ of the feedback power. For typical shock velocities, most of this energy will come out in ultraviolet and optical emission lines. The energy in the optical lines will be greater than the UV lines by $\sim 20 \%$, suggesting that $\sim 15 \%$ of the feedback power will come out in optical emission (Shull \& McKee 1979).

For objects that show outflow in emission, we estimate the energy injection rate into the gas from a starburst by using the SFR with a Salpeter IMF. We scale the predicted supernova energy injection rates (Leitherer et al. 1999; SB99) to the calculated SFR for each source, after correcting for differences in the assumed IMF low-mass cutoff (Kennicutt 0.1-100 $M_{\odot}$ and SB99 1-100 $M_{\odot}$ ). This estimate assumes a continuous star formation model over a timescale longer than $40 \mathrm{Myr}$ - producing a nearly continuous injection of feedback energy via supernovae and stellar winds.
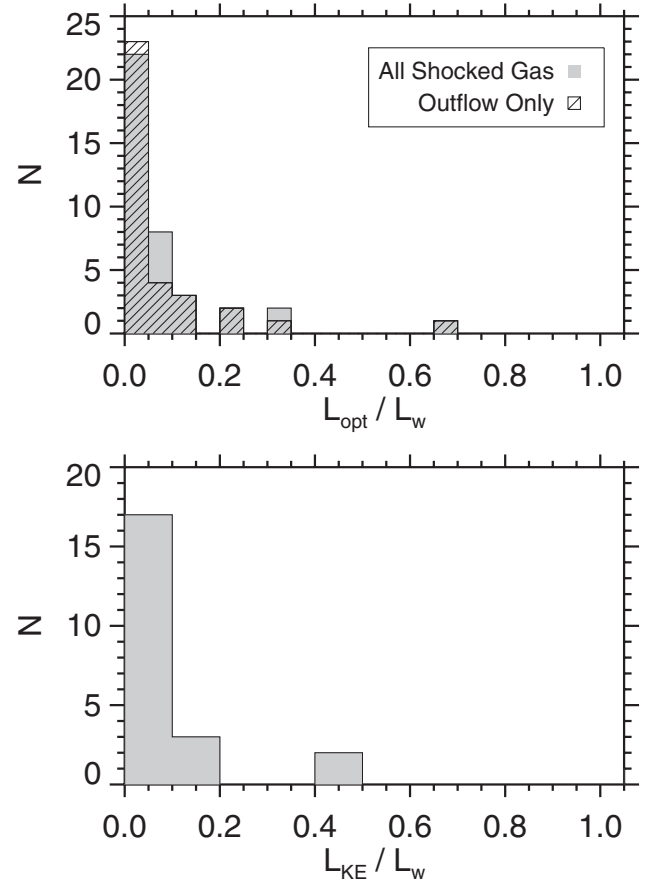

Figure 6. Top: a histogram showing the measured luminosity $\left(L_{\mathrm{opt}}\right)$ emitted in shocked gas compared to the mechanical energy injection rate $\left(L_{w}\right)$ from supernovae scaled from the IR star formation rate with SB99. For a whole galaxy, both the emission associated with the shocked outflow (hashed) and the emission from all shocked regions (solid) are generally in the range 0\%-15\%, with the peak below $5 \%$. The measured values are consistent with Weaver et al. (1977). Bottom: the fraction of the feedback energy that contributes the kinetic energy in the mass outflow is again consistent with the Weaver model.

Using our estimates of physical properties in Section 4.2.1 and the shock speeds from Paper I, we compare measured energies to this simple model. In Figure 6, we show the ratio of the total optical luminosity $\left(L_{\text {opt }}\right)$ of shocked outflowing gas in the measured lines compared to the mechanical power $\left(L_{w}\right.$, inferred from the SFR). Since this ratio generally falls short of the predicted $15 \%$, it follows that supernova feedback is a plausible source of power for this shocked emission for most of the sample. However, optical emission line luminosity for four of the galaxies (IRAS 05246+0103, IRAS 09583+4714, IRAS 13451+1232, and IRAS 20087-0308) exceeds the expected fraction of energy injected into outflow by supernovae. The excess emission in three objects (IRAS 05246+0103, IRAS 09583+4714, and IRAS $13451+1232$ ) may be due to additional emission from an AGN NLR since for these three objects the emission region size from scaling relations is consistent with that of an AGN NLR. In IRAS 20087-0308, the emission encounters heavy extinction that makes the Balmer decrement difficult to measure, resulting in a possible overcorrection to the extinction, which would then skew the $L_{\mathrm{opt}} / L_{\mathrm{w}}$ ratio.

We calculate the ratio of power that appears as kinetic energy in the outflow $\left(L_{\mathrm{KE}}\right)$ to the mechanical energy injection rate using estimated masses and velocities in Table 1 . This kinetic energy estimate uses $\dot{M}_{\mathrm{L}}$ as the mass outflow rate and $v_{\text {out }}$ for the velocity. In Figure 6, we show that this energy injection rate is again consistent with the fraction of energy in outflow models (average $L_{\mathrm{KE}} / L_{\mathrm{W}}=0.08$ ), which predict a $\sim 20 \%$ fraction of mechanical energy in the kinetic energy of the shell. The correspondence between the kinetic energy fraction $\left(L_{\mathrm{KE}} / L_{\mathrm{W}}\right)$ and the model predictions suggests that supernova winds are at 
work driving the outflows in ULIRGs. It also implies that the estimated masses are correct within an order of magnitude.

\section{DISCUSSION}

\subsection{Outflows in Integrated Spectra}

In observations of galaxies at high redshift, obtaining similar spatial resolution to this study is difficult due to the small angular size of galaxies. As a result, most studies use integrated spectra; however, the degree to which spatially integrated emission lines show evidence for the outflows is unclear. Using the present data set, we can still detect the presence of outflows, even in integrated spectra.

\subsubsection{Spectral Classifications from the Total Line Flux}

For low spectral resolution, spatially unresolved studies of galaxies, one means of examining the excitation mechanism is through total line flux classifications. We examine the relation between classification and the presence of shocks to determine which of the Seyferts, LINERs, and H II-classified galaxies host shocked gas.

We start by classifying the ULIRGs in our sample based solely on their integrated spectra obtained by integrating along the slit (Paper I). Though these classifications do not consider the effects of slit loss, the extended shocked gas that we have identified in the resolved analysis will tend to move the classification out of the $\mathrm{H}$ II class and into the Seyfert and LINER classifications. All six galaxies classified as Seyferts (Paper I, Table 2) present broad, blueshifted, shock-excited outflows in the kpc-scale apertures. The narrow emission line components in these Seyferts also present shock-like ratios in four out of six cases-IRAS 00188-0856, IRAS 01003-2238, IRAS $13451+1232$, and IRAS 15130-1958. The luminosity $\left.\left(L_{[\mathrm{O}} \mathrm{II}\right]\right)$ of the broad, shocked components suggests a spatially resolved NLR, allowing us to conclude that AGN photoionization is the source of excitation for these four galaxies. In contrast, in the Seyfert galaxies IRAS 09583+4714 and IRAS 12071-0444, the broad components and the narrow components differ in excitation. Of the two galaxies in IRAS $09583+4714$, the galaxy with shocked outflow presents narrow component excitation consistent with $\mathrm{H}$ II and has a strong, disk-like rotation gradient. In IRAS 12071-0444, the highly extended $( \pm 9 \mathrm{kpc})$ narrow components have excitation more consistent with LINERs. For both of these exceptional galaxies, scaling relations from $L_{\left[\mathrm{O}_{\text {III }}\right]}$ suggest that the outflow region is larger than what would be expected from an AGN NLR. The inconsistency of the outflow excitation with the integrated classification in these two galaxies suggests that some ULIRGs may be misclassified as Seyfert galaxies, when they instead host shocked outflows driven by star formation.

The subset of nine galaxies classified as LINERs frequently hosts shocks in both narrow and broad components. In all but one of these objects, narrow, shocked emission appears at least $5 \mathrm{kpc}$ from the associated nucleus. In five of the six LINERs that show evidence for outflows, the blueshifted wind signature extends several kpc beyond the nucleus. None of the nine galaxies have sufficient $L_{\left[\mathrm{O}_{\text {III }}\right]}$ to suggest that the shocked outflow or the narrow, shocked component is powered by an AGN NLR. These examples emphasize that an integrated galaxy spectrum with a LINER classification need not indicate the presence of an AGN.

Among the sample of 48 galaxies, 12 have emission-line ratios on the borderline between classifications. Six of these borderline galaxies have ratios between the Seyfert and LINER classifications and have broad outflows and extended shocks that are not part of an outflow. The two borderline galaxies with classifications between $\mathrm{H}$ II and LINER do not host shocked outflows but do have extended shocks, responsible for moving the net classification slightly outside the range of the $\mathrm{H}$ II galaxies. The remaining four galaxies are a mix of classifications, without a clear trend in behavior.

The largest fraction of the sample (21 out of 48 galaxies) is classified as $\mathrm{H}$ II in the integrated spectra. These galaxies share the same $\mathrm{H}$ II classification in the diagnostic diagrams that compare $[\mathrm{O} \mathrm{I}] / \mathrm{Ha}$ and $[\mathrm{S} \mathrm{II}] / \mathrm{H} \alpha$, but the $[\mathrm{N} \mathrm{II}] / \mathrm{H} \alpha$ can be either H II or "composite" as described in Kewley et al. (2006). The subset of galaxies with a "composite" classification hosts either shocked outflows or shocks in the extended narrow emission line gas. Clearly, the presence of shocks in these objects modifies the $[\mathrm{N}$ II $] / \mathrm{H} \alpha$ classification, and observations with kpc-scale spatial resolution show that supernovae are a plausible source of the forbidden-line enhancement and the outflow.

Emission components with shock-like excitation appear in ULIRGs of all classification types. In Seyfert galaxies, shockexcited regions often have broad widths consistent with outflowing gas but do not necessarily indicate an AGN-driven outflow. In the LINERs, the overall classification is influenced by the contribution of the shock-like emission in non-nuclear regions. For $\mathrm{H}$ II galaxies, the contribution of shock-like emission is more challenging to see in integrated spectra. The contribution of a shock region can clearly modify the position on the diagnostic diagram and even for the $\mathrm{H}$ II galaxies. The contribution of components will also influence our interpretation of other parameters such as metallicity or AGN contribution, as is the case for the "composite" classified galaxies.

\subsubsection{Identifying Shocks in the Integrated Spectral Profiles}

Our analysis of spatially and spectrally resolved line emission provides new insight on how to identify regions of shocked gas. Here, we examine whether the method works on integrated spectra with moderate spectral resolution. This analysis differs from the previous section by comparing the line shapes in the integrated profiles to those in individual spatial apertures.

This analysis allows us to define the required ranges and resolutions of instruments obtaining the integrated spectra. Clearly, the integrated spectra must cover $[\mathrm{O} \mathrm{I}] \lambda 6300$. The strong [O I] $\lambda 6300$ emission from shocked regions and the separation of this transition from other lines make it critical for uniquely fitting multiple velocity components. Furthermore, we will assume that the integrated spectra have spectral resolution of at least $R \geqslant 5000$ and signal-to-noise ratio $\sim 15$ if multicomponent fitting will be attempted.

Of the 24 galaxies that exhibit broad blueshifted outflow in the spatially resolved analysis, 19 galaxies also show these features in the integrated spectrum. In the remaining 5 galaxies, the strong narrow lines observed in the resolved analysis mask the signature of broad outflow in the integrated spectrum.

Regions with shock-like narrow $\left(\sigma_{v}<150 \mathrm{~km} \mathrm{~s}^{-1}\right)$ emission lines appear frequently (34 of 48 galaxies) in the spatially resolved analysis. Over a large range in position, these narrow features are identified at Doppler shifts ranging from $-250 \mathrm{~km} \mathrm{~s}^{-1}$ to $250 \mathrm{~km} \mathrm{~s}^{-1}$. Averaging components with a large range in Doppler shift act to broaden the integrated line profiles, which is the case for 5 of the 34 galaxies. The summed components with narrow shock-like emission form an emission line of width $\sigma_{v}>150 \mathrm{~km} \mathrm{~s}^{-1}$ in the integrated spectrum. These galaxies 

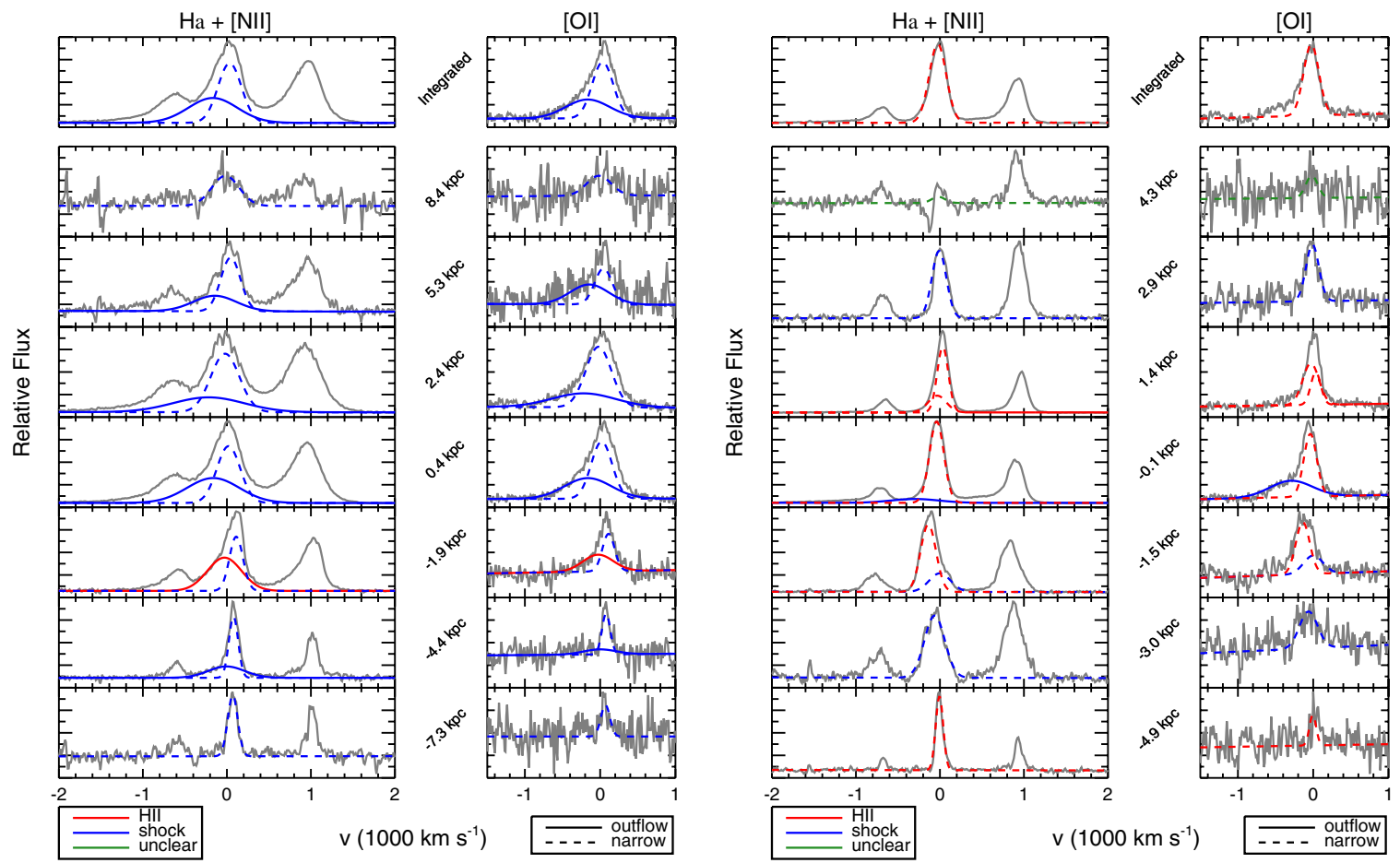

Figure 7. Plot of the $\mathrm{H} \alpha+[\mathrm{N} \mathrm{II}]$ and $\left[\mathrm{O}_{\mathrm{I}}\right]$ regions of the spectrum for both the integrated apertures and the spatially resolved apertures for IRAS $16090-0139$ and IRAS 23365+3604. Line color represents the excitation classification of each component into "H II" (red), "Shock-like" (blue), and "unclear" (green). The line style identified the components that are part of an identified outflow (solid), compared to the rest of the emission components (dashed). Left: IRAS 16090-0139 presents outflow components in the resolved analysis that also appears in the integrated spectrum. The observed narrow shock also appears in the integrated profile fit. Right: in the nuclear aperture of IRAS $23365+3604$ we identify outflow from the kinematics in the emission component. The apertures at $2.9 \mathrm{kpc}$ and $3 \mathrm{kpc}$ from the continuum source identify emission from shocked gas. All of these features are washed out in the integrated profile.

are mostly classified as $\mathrm{H}$ II with flux ratios in the "composite" region of the $[\mathrm{N} \mathrm{II}] / \mathrm{H} \alpha$ versus $[\mathrm{O} \mathrm{III}] / \mathrm{H} \beta$ diagnostic diagram. Figure 7 shows the integrated aperture and spatially resolved apertures for IRAS 16090-0139, which exhibits the outflow and narrow shocks from the resolved analysis in the integrated profile. We also compare this to the case of IRAS 23365+3604, which shows both types of shock in the spatially resolved analysis, while the integrated case is only identified as H II. In 15 out of the 34 galaxies, the Doppler shifts of the narrow, shocked components span a small enough range to be detected as narrow, shocked components in the integrated spectrum. A narrow span of Doppler shifts would also make any broad outflow more identifiable, which means that objects with low-inclination gas disks could show broad outflows more often.

We conclude that applying the multiple component fitting technique to integrated spectra would have some success in identifying broad, shocked components from outflows. In our sample, the outflow is undetected in the integrated spectrum only $20 \%$ of the time. In these five failures, a broad component is in fact detected in the integrated spectrum, but the spatially resolved analysis indicates that the emission comes from a gas rotating like a disk. Building a better understanding of the physical origin of the narrow, shock-like emission component is therefore critical for interpreting the presence of broad, shocked emission in integrated spectra.

\subsection{Lessons from the Local Laboratories}

While there are important differences between ULIRGs and star-forming disks at $z>2$, ULIRGs present high specific SFRs similar to the high end of star-forming galaxies from $2.5<z<5$
(Elbaz et al. 2007; Cunha et al. 2010; Feulner et al. 2005). The similar specific SFRs suggest that local ULIRGs can provide insight into processes that occur below current resolution limits and that the broad emission components may share a common origin. Using adaptive optics spectroscopy of $z \sim 2$ galaxies, star-forming clumps were recently shown to be the origin of the broad $\mathrm{H} \alpha /[\mathrm{N}$ II $]$ line wings (Genzel et al. 2011). The $\sim 4 \mathrm{kpc}$ offset of these clumps from the galactic center suggests that a supernova-driven outflow, rather than an AGN, broadens the line emission. The more central location of the broad emission found in the ULIRGs illustrates that they are not direct analogs of the $z \sim 2$ galaxies, but the radius and luminosity of the broad emission region in most of these ULIRGs are uncomfortably large to be caused by the AGN.

In the echellete spectra, simultaneous fits of multiple transitions identify broad emission features in the line profiles. Our fitted line widths are not as large as the broad $\mathrm{H} \alpha /[\mathrm{N} \mathrm{II}]$ wings reported for $z \sim 2$ galaxies (Shapiro et al. 2009) because our spectra require broad components in the forbidden lines and the recombination lines. Additionally, since ULIRGs are known to be much more reddened than the $z \sim 2$ starbursts, broad emission line profiles produced by similar physical processes in the less dusty $z \sim 2$ galaxies will be more symmetric with respect to the systemic velocity and therefore broader than those in ULIRGs. While Shapiro et al. (2009) had difficulty distinguishing BLRs and superwinds as the source of the broad $\mathrm{H} \alpha$ emission in stacks of $z \sim 2$ spectra, a simultaneous fit of unblended forbidden lines such as [O I] and [S II] along with the Balmer lines assists in identification of the actual width and excitation mechanism of $\mathrm{H} \alpha$ in the $\mathrm{H} \alpha+[\mathrm{N}$ II] line profile. 
The estimates of outflow mass and outflow rate in the local ULIRGs provide context for the estimates of outflow rate and mass in the star-forming clumps at $z \sim 2$ (Genzel et al. 2011). The SFRs in these ULIRGs $\left(24-180 M_{\odot} \mathrm{yr}^{-1}\right)$ are much greater than the SFRs in the $z \sim 2$ star-forming clumps (3.3-40 $\left.M_{\odot} \mathrm{yr}^{-1}\right)$, but our estimates of the mass outflow rate are much lower (0.4-58 $M_{\odot} \mathrm{yr}^{-1}$ for ULIRGs and 6-200 $M_{\odot} \mathrm{yr}^{-1}$ for star-forming clumps). These results are consistent with a higher $\eta$ for warm ionized gas at high redshift and come from assuming the same $n_{e}$ for both galaxy populations. As an alternative interpretation, if one demands $\eta$ be the same, then $n_{e}$ would be a factor of 10 higher at high $z$.

Some properties of these outflows are easier to study in nearby galaxies, and the methods in this study illustrate how more information about the outflows at $z \sim 2$ can be obtained, even in integrated spectra. As mentioned already, for example, the outflows in $z \sim 2$ galaxies likely have shock-like line ratios similar to those in our ULIRG sample. The properties of these shocks can be estimated once spectral coverage of additional lines-particularly [O I], $\mathrm{H} \beta$, and [O III] - is obtained at moderate spectral resolution, $R \approx 5000$, with similar signalto-noise ratio $(\sim 15)$ as in this study.

\section{SUMMARY}

Paper I mapped optical, emission-line ratios across 39 ULIRGs in two dimensions, i.e., line-of-sight velocity plus one spatial dimension. In this paper, we have used those measurements to describe the kinematics of regions excited by different physical processes. Figure 2 shows the distribution of line-ofsight velocity dispersion for regions with shock-like and $\mathrm{H}$ II-like line ratios. The median linewidth for the shock-like components, $\sigma_{v} \approx 144 \mathrm{~km} \mathrm{~s}^{-1}$, is higher than that of the $\mathrm{H}$ II-like components, $61 \mathrm{~km} \mathrm{~s}^{-1}$. The difference is caused by the large number of shock-like components broader than $\sigma_{v} \approx 150 \mathrm{~km} \mathrm{~s}^{-1}$ and the near absence of $\mathrm{H}$ II-like components with linewidths this large.

These broad emission components are relevant to our understanding of gas inflows and outflows because gas in virial equilibrium would produce lines with smaller widths. Our results provide insight into the interpretation of the emission-line spectra of high-redshift galaxies, particularly dusty galaxies with extremely high SFRs.

We find that the broad shock-like components are typically spatially extended, reaching radii up to $6 \mathrm{kpc}$ in Figure 4. We show that in 17 out of 24 galaxies that show evidence for an outflow with broad blueshifted emission components, the size of this region exceeds that expected for an AGN NLR under the assumption that $[\mathrm{O}$ III] $\lambda 5007$ luminosity from components with $\log ([\mathrm{O} \mathrm{III}] / \mathrm{H} \beta)>0.5$ is powered by the AGN. We estimate the total power radiated by summing the optical luminosities of the broad-line components and applying a geometrical correction for slit losses. Since the resulting shock luminosities range from $5 \%$ to $20 \%$ of the mechanical power from supernova explosions (similar to model predictions), we conclude that supernovae are a viable power source for the gas flows that generate the shocks.

In ULIRGs, dust absorbs much of the starlight, and the centers of these galaxies are not transparent at ultraviolet and optical wavelengths. At least in the central few kpc, we can be reasonably certain that the emergent emission-line profile is shaped by gas on the near side of the galaxy. We therefore interpret the blueshifts of the broad components as direct evidence that the gas is outflowing. In less dusty galaxies, this outflow component would presumably also have a red wing from emission coming from the far side of the galaxy.

The broad components have surprisingly smooth line profiles. Across luminous infrared galaxies with winds, for example, the $\mathrm{H} \alpha+\left[\begin{array}{ll}\mathrm{N} & \mathrm{II}\end{array}\right]$ profiles are well described by a single, Gaussian component where emission from $\mathrm{H}$ II regions in the underlying disk dominates the flux. When the outflows are observed against the sky, along the minor axis, the emission lines are typically double peaked (Lehnert \& Heckman 1995). The relative intensities of the two components have been shown to reflect the inclination and opening angle of a biconical outflow (Heckman et al. 1990). That some of the broad emission is detected beyond the continuum emission in these ULIRGs, yet does not have a double-peaked profile, may be related in part to the obscuration of the back side of the outflow. However, it is not obvious that the galaxies are opaque at these large radii, and we tentatively conclude that the warm ionized, outflowing gas in ULIRGs does not share the biconical structure that describes outflows in LIRGs very well.

The prominence of shock-excited emission in these starburst galaxies raises questions about how shocked emission skews the emission-line ratios measured from integrated spectra. Since the luminosity in the ultraviolet, ionizing continuum exceeds the mechanical power from supernova and stellar winds by about an order of magnitude (Leitherer et al. 1999; Martin 2007), we would not expect shocks from galactic winds to determine the line ratios measured in integrated spectra. What Figure 7 clearly demonstrates, however, is that when integrated spectra have moderately high spectral resolution $(R \sim 5000)$, shocked, outflowing gas can sometimes be recognized by tying the kinematics of the blended Balmer lines to those of the forbidden lines. In our case, the use of $[\mathrm{O}$ I] $\lambda 6300$ proved vital in deblending the emission-line profiles, since it is well separated from another emission feature, allowing us to deblend the $\mathrm{H} \alpha+$ $[\mathrm{N}$ II] profile. In future studies, the measurement of the shocksensitive [O I] line will provide another means for identifying shocked gas. Further, the use of simultaneous multi-component line fitting of $\mathrm{H} \alpha,[\mathrm{N}$ II $],[\mathrm{S} \mathrm{II}],\left[\mathrm{O}_{\mathrm{I}}\right], \mathrm{H} \beta$, and [O III] in the rest-frame optical spectrum can provide useful diagnostics of shock velocities and radiative energy losses in galaxies over a very broad range of cosmic time.

The authors thank Kristian Finlator, Nicolas Bouché, Alaina Henry, Vardha Bennert, Tommaso Treu, and Omer Blaes for illuminating discussions. This work was supported by the National Science Foundation under contracts AST-0808161 and AST-1109288 and the Department of Education through the Graduate Assistance in Areas of National Need program. A portion of this work was completed at the Aspen Center for Physics. The authors wish to recognize and acknowledge the very significant cultural role and reverence that the summit of Mauna Kea has always had within the indigenous Hawaiian community. We are most fortunate to have the opportunity to conduct observations from this mountain.

Facility: Keck:II

\section{REFERENCES}

Armus, L., Bernard-Salas, J., Spoon, H. W. W., et al. 2006, ApJ, 640, 204 Armus, L., Charmandaris, V., Bernard-Salas, J., et al. 2007, ApJ, 656, 148 Armus, L., Charmandaris, V., Spoon, H. W. W., et al. 2004, ApJS, 154, 178 Armus, L., Heckman, T. M., \& Miley, G. K. 1990, ApJ, 364, 471

Bennert, N., Falcke, H., Schulz, H., Wilson, A. S., \& Wills, B. J. 2002, ApJ, 574, L105 
Bennert, N., Jungwiert, B., Komossa, S., Haas, M., \& Chini, R. 2006a, A\&A, 459,55

Bennert, N., Jungwiert, B., Komossa, S., Haas, M., \& Chini, R. 2006b, A\&A, 456, 953

Bentz, M. C., Walsh, J. L., Barth, A. J., et al. 2009, ApJ, 705, 199

Borne, K. D., Bushouse, H., Colina, L., et al. 1999, Ap\&SS, 266, 137

Brewer, B. J., Treu, T., Pancoast, A., et al. 2011, ApJ, 733, L33

Calzetti, D., Kinney, A. L., \& Storchi-Bergmann, T. 1994, ApJ, 429, 582

Cardelli, J. A., Clayton, G. C., \& Mathis, J. S. 1989, ApJ, 345, 245

Cunha, E. D., Charmandaris, V., Díaz-Santos, T., et al. 2010, A\&A, 523, 78

Daddi, E., Dickinson, M., Morrison, G., et al. 2007, ApJ, 670, 156

Dasyra, K. M., Tacconi, L. J., Davies, R. I., et al. 2006, ApJ, 638, 745

Dietrich, M., Wagner, S. J., Courvoisier, T. J.-L., Bock, H., \& North, P. 1999, A\&A, 351, 31

Dopita, M. A., \& Sutherland, R. S. 1995, ApJ, 455, 468

Elbaz, D., Daddi, E., Borgne, D. L., et al. 2007, A\&A, 468, 33

Feulner, G., Gabasch, A., Salvato, M., et al. 2005, ApJ, 633, L9

Genzel, R., Newman, S., Jones, T., et al. 2011, ApJ, 733, 101

Genzel, R., Tacconi, L. J., Rigopoulou, D., Lutz, D., \& Tecza, M. 2001, ApJ, 563,527

Goerdt, T., Dekel, A., Sternberg, A., et al. 2010, MNRAS, 407, 613

Gonçalves, T. S., Basu-Zych, A., Overzier, R., et al. 2010, ApJ, 724, 1373

Greene, J. E., Zakamska, N. L., Ho, L. C., \& Barth, A. J. 2011, ApJ, 732, 9

Heckman, T. M., Armus, L., \& Miley, G. K. 1987, AJ, 93, 276

Heckman, T. M., Armus, L., \& Miley, G. K. 1990, ApJS, 74, 833

Heckman, T. M., Borthakur, S., Overzier, R., et al. 2011, ApJ, 730, 5

Hopkins, P. F. 2012, MNRAS, 420, L8

Hopkins, P. F., Hernquist, L., Cox, T. J., Dutta, S. N., \& Rothberg, B. 2008, ApJ, 679,156

Hopkins, P. F., Hernquist, L., Cox, T. J., et al. 2005, ApJ, 630, 705

Hopkins, P. F., Keres, D., Murray, N., Quataert, E., \& Hernquist, L. 2011, arXiv: 1111.6591

Kauffmann, G., Heckman, T. M., Tremonti, C., et al. 2003, MNRAS, 346, 1055

Kaufman, M., Bash, F. N., Kennicutt, R. C., \& Hodge, P. W. 1987, ApJ, 319, 61

Kennicutt, R. C. 1984, ApJ, 287, 116

Kennicutt, R. C. 1998, ApJ, 498, 541

Kewley, L. J., Dopita, M. A., Sutherland, R. S., Heisler, C. A., \& Trevena, J. 2001, ApJ, 556, 121

Kewley, L. J., Groves, B., Kauffmann, G., \& Heckman, T. 2006, MNRAS, 372 , 961

Law, D. R., Steidel, C. C., Erb, D. K., et al. 2009, ApJ, 697, 2057

Lehnert, M. D., \& Heckman, T. M. 1995, ApJS, 97, 89
Leitherer, C., Schaerer, D., Goldader, J. D., et al. 1999, ApJS, 123, 3

Martin, C. L. 2005, ApJ, 621, 227

Martin, C. L. 2006, ApJ, 647, 222

Martin, C. L. 2007, in IAU Symp. 235, Galaxy Evolution across the Hubble Time, ed. F. Combes \& J. Palous (Cambridge: Cambridge University Press), 280

Monreal-Ibero, A., Arribas, S., \& Colina, L. 2006, ApJ, 637, 138

Murphy, T. W., Armus, L., Matthews, K., et al. 1996, AJ, 111, 1025

Murray, N., Martin, C. L., Quataert, E., \& Thompson, T. A. 2007, ApJ, 660, 211

Murray, N., Quataert, E., \& Thompson, T. A. 2005, ApJ, 618, 569

Noguchi, M. 1999, ApJ, 514, 77

Osterbrock, D. E., \& Ferland, G. J. 2006, in Astrophysics of Gaseous Nebulae and Active Galactic Nuclei (2nd. ed; Sausalito, CA: Univ. Science Books)

Overzier, R. A., Heckman, T. M., Kauffmann, G., et al. 2008, ApJ, 677, 37

Overzier, R. A., Heckman, T. M., Tremonti, C., et al. 2009a, ApJ, 706, 203

Overzier, R. A., Heckman, T. M., Wang, J., et al. 2011, ApJ, 726, L7

Overzier, R. A., Shu, X., Zheng, W., et al. 2009b, ApJ, 704, 548

Peterson, B. M. 2006, in Physics of Active Galactic Nuclei at All Scales, ed. D. Alloin, R. Johnson, \& P. Lira, (Lecture Notes in Physics, Vol. 693), 77

Rich, J. A., Kewley, L. J., \& Dopita, M. A. 2011, ApJ, 734, 87

Rupke, D. S., \& Veilleux, S. 2005, ApJ, 631, L37

Rupke, D. S., Veilleux, S., \& Sanders, D. B. 2002, ApJ, 570, 588

Rupke, D. S., Veilleux, S., \& Sanders, D. B. 2005a, ApJ, 632, 751

Rupke, D. S., Veilleux, S., \& Sanders, D. B. 2005b, ApJS, 160, 87

Rupke, D. S., Veilleux, S., \& Sanders, D. B. 2005c, ApJS, 160, 115

Sanders, D. B., Scoville, N. Z., Young, J. S., et al. 1986, ApJ, 305, L45

Schlegel, D. J., Finkbeiner, D. P., \& Davis, M. 1998, ApJ, 500, 525

Schreiber, N. M. F., Genzel, R., Lehnert, M. D., et al. 2006, ApJ, 645, 1062

Searle, L. 1971, ApJ, 168, 327

Shapiro, K. L., Genzel, R., Förster Schreiber, N. M., et al. 2008, ApJ, 682, 231

Shapiro, K. L., Genzel, R., Quataert, E., et al. 2009, ApJ, 701, 955

Shull, J. M., \& McKee, C. F. 1979, ApJ, 227, 131

Soto, K. T., \& Martin, C. L. 2012, arXiv:1205.0082

Spoon, H. W. W., \& Holt, J. 2009, ApJ, 702, L42

Springel, V., Matteo, T. D., \& Hernquist, L. 2005, MNRAS, 361, 776

Toomre, A., \& Toomre, J. 1972, ApJ, 178, 623

Veilleux, S., Rupke, D. S. N., Kim, D.-C., et al. 2009, ApJS, 182, 628

Weaver, R., Mccray, R., Castor, J., Shapiro, P., \& Moore, R. 1977, ApJ, 218, 377

Westmoquette, M., Clements, D., Bendo, G., \& Khan, S. 2012, MNRAS, 424, 416 